\title{
Existence of solution for stochastic differential equations driven by $G$-Lévy process with discontinuous coefficients
}

\author{
Bingjun Wang ${ }^{1,2^{*}}$ and Mingxia Yuan ${ }^{3}$
}

${ }^{*}$ Correspondence:
wbj586@126.com.cn
'School of Mathematical Science,
Nanjing Normal University, Nanjing,
210023, P.R. China
${ }^{2}$ Jinling Institute of Technology,
Nanjing, 211169, P.R. China
Full list of author information is
available at the end of the article

available at the end of the article

\begin{abstract}
The existence theory for the vector-valued stochastic differential equations driven by G-Brownian motion and pure jump G-Lévy process (G-SDEs) of the type $d Y_{t}=f\left(t, Y_{t}\right) d t+g_{j, k}\left(t, Y_{t}\right) d\left\langle B^{j}, B^{k}\right\rangle_{t}+\sigma_{i}\left(t, Y_{t}\right) d B_{t}^{i}+\int_{R_{0}^{d}} K\left(t, Y_{t}, z\right) L(d t, d z), t \in[0, T]$, with first two and last discontinuous coefficients, is established. It is shown that the G-SDEs have more than one solution if the coefficients $f, g, K$ are discontinuous functions. The upper and lower solution method is used.
\end{abstract}

MSC: $60 \mathrm{H} 05 ; 60 \mathrm{H} 10 ; 60 \mathrm{H} 20$

Keywords: stochastic differential equations; G-Lévy process; upper and lower solution; discontinuous coefficients

\section{Introduction}

In recent years much effort has been made to develop the theory of sublinear expectations connected with the volatility uncertainty and the so-called G-Brownian motion. GBrownian motion was introduced by Shige Peng in $[1,2]$ as a way to incorporate the unknown volatility into financial models. Its theory is tightly associated with the uncertainty problems involving an undominated family of probability measures. Soon other connections have been discovered, not only in the field of financial mathematics, but also in the theory of path-dependent partial differential equations or backward stochastic differential equations. Thus $G$-Brownian motion and connected $G$-expectation are attractive mathematical objects.

Returning, however, to the original problem of volatility uncertainty in the financial models, one feels that $G$-Brownian motion is not sufficient to model the financial world, as both $G$ - and the standard Brownian motion share the same property, which makes them often unsuitable for modeling, namely, the continuity of paths. Therefore, it is not surprising that $\mathrm{Hu}$ and Peng [3] introduced the process with jumps, which they called G-Lévy process. Then Ren [4] introduced the representation of the sublinear expectation as an upper-expectation. In [5], the author concentrated on establishing the integration theory for G-Lévy process with finite activity, introduced the integral w.r.t. the jump measure associated with the pure jump G-Lévy process and gave the Itô formula for general G-Itô Lévy process. 
Under the integration theory for G-Lévy process, Paczka [5] established the existence and uniqueness of solutions for the following stochastic differential equation driven by $G$ Brownian motion and pure jump G-Lévy process with Lipschitz continuous coefficients:

$$
\begin{aligned}
Y_{t}= & Y_{0}+\int_{0}^{t} f\left(s, Y_{s}\right) d s+\int_{0}^{t} g_{j, k}\left(s, Y_{s}\right) d\left\langle B^{j}, B^{k}\right\rangle_{s} \\
& +\int_{0}^{t} \sigma_{i}\left(s, Y_{s}\right) d B_{s}^{i}+\int_{0}^{t} \int_{R_{0}^{d}} K\left(s, Y_{s}, z\right) L(d s, d z),
\end{aligned}
$$

where $Y_{0} \in R^{n},\left(\left\langle B^{j}, B^{k}\right\rangle_{t}\right)_{t \geq 0}$ is the mutual variation process of the G-Brownian motion $\left(B_{t}\right)_{t \geq 0}, L(t, z)$ is pure jump G-Lévy process. For each $x \in R^{n}$, the coefficients $f(t, x), g_{j, k}(t, x), \sigma_{i}(t, x)$ are in the space $\hat{M}_{G}^{2}\left(0, T ; R^{n}\right), K(t, x, z) \in \hat{H}_{G}^{2}\left([0, T] \times R_{0}^{d} ; R^{n}\right)$ (which will be introduced in Section 2). A process $Y_{t}$ belonging to $\hat{M}_{G}^{2}\left(0, T ; R^{n}\right)$ and satisfying G-SDE (1.1) is said to be its solution.

Motivated by the importance of discontinuous functions, Faizullah and Piao [6] established the existence of solutions for the stochastic differential equations driven by GBrownian motion with a discontinuous drift coefficient. Then Faizullah [7] developed the existence theory when the coefficient $f$ or the coefficients $f$ and $g$ simultaneously are discontinuous functions. Motivated by the aforementioned works, in this paper, we consider equation (1.1) and assume that $f(t, x), g(t, x)$ and $K(t, x, z)$ are discontinuous for all $x \in R^{n}$.

The rest of this paper is organized as follows. In Section 2, we introduce some preliminaries. In Section 3, the existence of solutions for G-SDE (1.1) with simultaneous discontinuous coefficients $f, g$ and $K$ is developed.

\section{Preliminaries}

In this section, we introduce some notations and preliminary results in G-framework which are needed in the sequence. More details can be found in [5, 8-13].

Definition 2.1 Let $\Omega$ be a given set, and let $\mathcal{H}$ be a linear space of real-valued functions defined on $\Omega$. Moreover, if $X_{i} \in \mathcal{H}, i=1,2, \ldots, d$, then $\varphi\left(X_{1}, \ldots, X_{d}\right) \in \mathcal{H}$ for all $\varphi \in C_{b \text {,lip }}\left(R^{d}\right)$, where $C_{b \text {,lip }}\left(R^{d}\right)$ is the space of all bounded real-valued Lipschitz continuous functions. A sublinear expectation $\mathbb{E}$ is a functional $\mathbb{E}: \mathcal{H} \rightarrow R$ satisfying the following properties: for all $X, Y \in \mathcal{H}$, we have:

(i) Monotonicity: $\mathbb{E}[X] \geq \mathbb{E}[Y]$ if $X \geq Y$;

(ii) Constant preserving: $\mathbb{E}[C]=C$ for $C \in R$;

(iii) Sub-additivity: $\mathbb{E}[X+Y] \leq \mathbb{E}[X]+\mathbb{E}[Y]$;

(iv) Positive homogeneity: $\mathbb{E}[\lambda X]=\lambda \mathbb{E}[X]$ for $\lambda \geq 0$.

The triple $(\Omega, \mathcal{H}, \mathbb{E})$ is called a sublinear expectation space. $X \in \mathcal{H}$ is called a random variable in $(\Omega, \mathcal{H}, \mathbb{E})$. We often call $Y=\left(Y_{1}, \ldots, Y_{d}\right), Y_{i} \in \mathcal{H}$ a $d$-dimensional random vector in $(\Omega, \mathcal{H}, \mathbb{E})$.

Definition 2.2 In a sublinear expectation space $(\Omega, \mathcal{H}, \mathbb{E})$, an $n$-dimensional random vector $Y=\left(Y_{1}, \ldots, Y_{n}\right)$ is said to be independent from an $m$-dimensional random vector $X=\left(X_{1}, \ldots, X_{m}\right)$ if for each $\varphi \in C_{b, \text { lip }}\left(R^{m+n}\right)$,

$$
\mathbb{E}[\varphi(X, Y)]=\mathbb{E}\left[\mathbb{E}[\varphi(x, Y)]_{x=X}\right] .
$$


Definition 2.3 Let $X_{1}, X_{2}$ be two $n$-dimensional random vectors defined on sublinear expectation spaces $\left(\Omega_{1}, \mathcal{H}_{1}, \mathbb{E}_{1}\right)$ and $\left(\Omega_{2}, \mathcal{H}_{2}, \mathbb{E}_{2}\right)$, respectively. They are called identically distributed, denoted by $X_{1} \stackrel{d}{=} X_{2}$, if

$$
\mathbb{E}_{1}\left[\varphi\left(X_{1}\right)\right]=\mathbb{E}_{2}\left[\varphi\left(X_{2}\right)\right], \quad \forall \varphi \in C_{b, \text { lip }}\left(R^{n}\right) .
$$

$\bar{X}$ is said to be an independent copy of $X$ if $\bar{X}$ is identically distributed with $X$ and independent of $X$.

Definition 2.4 (G-Lévy process) Let $X=\left(X_{t}\right)_{t \geq 0}$ be a $d$-dimensional càdlàg process on a sublinear expectation space $(\Omega, \mathcal{H}, \mathbb{E})$. We say that $X$ is a Lévy process if:

(i) $X_{0}=0$,

(ii) for each $s, t \geq 0$, the increment $X_{t+s}-X_{s}$ is independent of $\left(X_{t_{1}}, \ldots, X_{t_{n}}\right)$ for every $n \in N$ and every partition $0 \leq t_{1} \leq t_{2} \leq \cdots \leq t_{n} \leq s$,

(iii) the distribution of the increment $X_{t+s}-X_{s}, s, t \geq 0$ is stationary, i.e., does not depend on $s$.

Moreover, we say that a Lévy process $X$ is a G-Lévy process if it satisfies additionally the following conditions:

(iv) there is a $2 d$-dimensional Lévy process $\left(X_{t}^{c}, X_{t}^{d}\right)_{t \geq 0}$ such that for each $t \geq 0$,

$$
X_{t}=X_{t}^{c}+X_{t}^{d}
$$

(v) processes $X_{t}^{c}$ and $X_{t}^{d}$ satisfy the following conditions:

$$
\lim _{t \downarrow 0} \mathbb{E}\left[\left|X_{t}^{c}\right|^{3}\right] t^{-1}=0 ; \quad \mathbb{E}\left[\left|X_{t}^{d}\right|\right]<C t \quad \text { for all } t \geq 0
$$

Peng and Hu noticed in their paper that each G-Lévy process might be characterized by a non-local operator $G_{X}$.

Theorem 2.1 ([3]) Let $X$ be a G-Lévy process in $R^{d}$. For every $f \in C_{b}^{3}\left(R^{d}\right)$ such that $f(0)=0$, we put

$$
G_{X}[f(\cdot)]:=\lim _{\delta \downarrow 0} \mathbb{E}\left[f\left(X_{\delta}\right)\right] \delta^{-1}
$$

The above limit exists. Moreover, $G_{X}$ has the following Levy-Khintchine representation:

$$
G_{X}[f(\cdot)]=\sup _{(v, p, Q) \in \mathcal{U}}\left\{\int_{R_{0}^{d}} f(z) v(d z)+\langle D f(0), p\rangle+\frac{1}{2} \operatorname{tr}\left[D^{2} f(0) Q Q^{T}\right]\right\}
$$

where $R_{0}^{d}:=R^{d} \backslash\{0\}, \mathcal{U}$ is a subset $\mathcal{U} \subset \mathcal{V} \times R^{d} \times R^{d \times d}$ and $\mathcal{V}$ is a set of all Borel measures on $\left(R_{0}^{d}, \mathcal{B}\left(R_{0}^{d}\right)\right)$. We know additionally that $\mathcal{U}$ has the property

$$
\sup _{(v, p, Q) \in \mathcal{U}}\left\{\int_{R_{0}^{d}}|z| v(d z)+|p|+\operatorname{tr}\left[Q Q^{T}\right]\right\}<\infty .
$$

Theorem 2.2 ([3]) Let $X$ be a d-dimensional G-Lévy process. For each $\phi \in C_{b, \mathrm{lip}}\left(R^{d}\right)$, define $u(t, x):=\mathbb{E}\left[\phi\left(x+X_{t}\right)\right]$. Then $u$ is the unique viscosity solution of the following integro- 
PDE:

$$
\begin{aligned}
0= & \partial_{t} u(t, x)-G_{X}[u(t, x+\cdot)-u(t, x)] \\
= & \partial_{t} u(t, x)-\sup _{(v, p, Q) \in \mathcal{U}}\left\{\int_{R_{0}^{d}}[u(t, x+z)-u(t, x)] v(d z)\right. \\
& \left.+\langle D u(t, x), p\rangle+\frac{1}{2} \operatorname{tr}\left[D^{2} u(t, x) Q Q^{T}\right]\right\}
\end{aligned}
$$

with the initial condition $u(0, x)=\phi(x)$.

Theorem 2.3 Let $\mathcal{U}$ satisfy (2.1). Consider the canonical space $\Omega:=\mathbb{D}_{0}\left(R^{+}, R^{d}\right)$ of all càdlàg functions taking values in $R^{d}$ equipped with the Skorohod topology. Then there exists a sublinear expectation $\hat{\mathbb{E}}$ on $\mathbb{D}_{0}\left(R^{+}, R^{d}\right)$ such that the canonical process $\left(X_{t}\right)_{t \geq 0}$ is a G-Lévy process satisfying Levy-Khintchine representation with the same set $\mathcal{U}$.

The proof might be found in [3]. We will give, however, the construction of $\hat{\mathbb{E}}$ as it is important to understand it.

We denote $\Omega_{T}:=\left\{w_{\cdot \wedge T}: w \in \Omega\right\}$. Put

$$
\begin{aligned}
\operatorname{Lip}\left(\Omega_{T}\right):= & \left\{\xi \in L^{0}\left(\Omega_{T}\right): \xi=\phi\left(X_{t_{1}}, X_{t_{2}}-X_{t_{1}}, \ldots, X_{t_{n}}-X_{t_{n-1}}\right),\right. \\
& \left.\phi \in C_{b, \text { lip }}\left(R^{d \times n}\right), 0 \leq t_{1}<\cdots<t_{n}<T\right\},
\end{aligned}
$$

where $X_{t}(w)=w_{t}$ is the canonical process on the space $\mathbb{D}_{0}\left(R^{+}, R^{d}\right)$ and $L^{0}(\Omega)$ is the space of all random variables, which are measurable to the filtration generated by the canonical process. We also set

$$
\operatorname{Lip}(\Omega):=\bigcup_{T=1}^{\infty} \operatorname{Lip}\left(\Omega_{T}\right)
$$

Firstly, consider the random variable $\xi=\phi\left(X_{t+s}-X_{s}\right), \phi \in C_{b \text {,lip }}\left(R^{d}\right)$. We define

$$
\hat{\mathbb{E}}[\xi]:=u(s, 0)
$$

where $u$ is a unique viscosity solution of integro-PDE (2.2) with the initial condition $u(0, x)=\phi(x)$. For general

$$
\xi=\phi\left(X_{t_{1}}, X_{t_{2}}-X_{t_{1}}, \ldots, X_{t_{n}}-X_{t_{n-1}}\right), \quad \phi \in C_{b, \text { lip }}\left(R^{d \times n}\right),
$$

we set $\hat{\mathbb{E}}[\xi]:=\phi_{n}$, where $\phi_{n}$ is obtained via the following iterated procedure:

$$
\begin{aligned}
& \phi_{1}\left(x_{1}, \ldots, x_{n-1}\right)=\hat{\mathbb{E}}\left[\phi\left(x_{1}, \ldots, x_{n-1}, X_{t_{n}}-X_{t_{n-1}}\right)\right], \\
& \phi_{2}\left(x_{1}, \ldots, x_{n-2}\right)=\hat{\mathbb{E}}\left[\phi_{1}\left(x_{1}, \ldots, x_{n-2}, X_{t_{n-1}}-X_{t_{n-2}}\right)\right], \\
& \vdots \\
& \phi_{n-1}\left(x_{1}\right)=\hat{\mathbb{E}}\left[\phi_{n-2}\left(x_{1}, X_{t_{2}}-X_{t_{1}}\right)\right], \\
& \phi_{n}=\hat{\mathbb{E}}\left[\phi_{n-1}\left(X_{t_{1}}\right)\right] .
\end{aligned}
$$


Lastly, we extend the definition of $\hat{\mathbb{E}}$ on the completion of $\operatorname{Lip}\left(\Omega_{T}\right)($ respectively $\operatorname{Lip}(\Omega))$ under the norm $\|\cdot\|_{p}^{p}=\hat{\mathbb{E}}\left[|\cdot|^{p}\right], p \geq 1$. We denote such a completion by $L_{G}^{p}\left(\Omega_{T}\right)$ (or resp. $\left.L_{G}^{p}(\Omega)\right)$.

Let $\mathcal{B}(\Omega)$ be the Borel $\sigma$-algebra of $\Omega$. It was proved in [4] that there exists a weakly compact probability measure family $\mathcal{P}$ defined on $(\Omega, \mathcal{B}(\Omega))$ such that

$$
\hat{\mathbb{E}}[X]=\sup _{p \in \mathcal{P}} E_{P}[X], \quad \forall X \in L_{G}^{1}(\Omega),
$$

where $E_{P}$ is the linear expectation with respect to $P$.

Definition 2.5 We define the capacity $c$ associated with $\hat{\mathbb{E}}$ by putting

$$
c(A):=\sup _{p \in \mathcal{P}} P(A), \quad A \in \mathcal{B}(\Omega) .
$$

We will say that a set $A \in \mathcal{B}(\Omega)$ is polar if $c(A)=0$. We say that a property holds quasi-surely (q.s.) if it holds outside a polar set.

Remark 2.1 The condition (v) in Definition 2.4 implies that $X^{c}$ is a $d$-dimensional generalized G-Brownian motion and the pure jump part $X^{d}$ is of finite variation (see [3]). Moreover, $X^{c}$ is just the $d$-dimensional G-Brownian motion $B_{t}$ when $p=0$ in (2.1). In this paper, we always let $p=0$, i.e., the $G$-Lévy process $X$ consists of $G$-Brownian motion $B_{t}$ and the pure jump part.

Let $M_{G}^{0, p}(0, T)$ be the collection of processes of the following form: for a given partition $\left\{t_{0}, \ldots, t_{N}\right\}=\pi_{T}$ of $[0, T]$,

$$
\eta_{t}=\sum_{i=0}^{N-1} \xi_{i}(w) I_{\left[t_{i}, t_{i+1}\right)}(t)
$$

where $\xi_{i} \in L_{G}^{p}\left(\Omega_{t_{i}}\right), i=0,1, \ldots, N-1, p \geq 1$. For each $p \geq 1$, denote by $M_{G}^{p}(0, T)$ the completion of $M_{G}^{0, p}(0, T)$ under the norm $\|\eta\|_{M_{G}^{p}}:=\left(\hat{\mathbb{E}}\left[\int_{0}^{T}\left|\eta_{t}\right|^{p} d t\right]\right)^{\frac{1}{p}}$.

For each $\eta \in M_{G}^{p}(0, T), p \geq 2$, the $G$-Itô integral $\left\{\int_{0}^{t} \eta_{s} d B_{s}^{i}\right\}_{t \in[0, T]}$ is well defined. For each $\eta_{s}^{j, k} \in M_{G}^{p}(0, T), p \geq 1$, the integral $\left\{\int_{0}^{t} \eta_{s}^{j, k} d\left\langle B^{j}, B^{k}\right\rangle_{s}\right\}_{t \in[0, T]}$ is well defined. $i, j, k=1, \ldots, d$. See Peng [12] and Li et al. [13].

Lemma 2.1 ([7]) Let $\eta_{t}^{j, k}, \zeta_{t}^{j, k} \in M_{G}^{1}(0, T)$. If $\eta_{t}^{j, k} \leq \zeta_{t}^{j, k}$ for $t \in[0, T]$, then

$$
\int_{0}^{T} \eta_{t}^{j, k} d\left\langle B^{j}, B^{k}\right\rangle_{t} \leq \int_{0}^{T} \zeta_{t}^{j, k} d\left\langle B^{j}, B^{k}\right\rangle_{t}
$$

Assume that the G-Lévy process $X$ has finite activity, i.e.,

$$
\lambda:=\sup _{v \in \mathcal{V}} v\left(R_{0}^{d}\right)<\infty
$$


Let $X_{u-}$ denote the left limit of $X$ at point $u, \Delta X_{u}=X_{u}-X_{u-}$, then we can define a random measure $L(\cdot, \cdot)$ associated with the $G$-Lévy process $X$ by putting

$$
L(] s, t], A)=\sum_{s<u \leq t} \mathbb{I}_{A}\left(\Delta X_{u}\right), \quad \text { q.s. }
$$

for any $0<s<t<\infty$ and $A \in \mathcal{B}\left(R_{0}^{d}\right)$. The random measure is well defined and may be used to define the pathwise integral.

Let $H_{G}^{S}\left([0, T] \times R_{0}^{d}\right)$ be a space of all elementary random fields on $[0, T] \times R_{0}^{d}$ of the form

$$
K(u, z)(w)=\sum_{k=1}^{n-1} \sum_{l=1}^{m} F_{k, l}(w) \mathbb{I}_{]_{k}, t_{k+1}\right]}(u) \psi_{l}(z), \quad n, m \in \mathbb{N},
$$

where $0 \leq t_{1}<\cdots<t_{n} \leq T$ is the partition of $[0, T],\left\{\psi_{l}\right\}_{l=1}^{m} \subset C_{b, \text { lip }}\left(R^{d}\right)$ are functions with disjoint supports s.t. $\psi_{l}(0)=0$ and $F_{k, l}=\phi_{k, l}\left(X_{t_{1}}, \ldots, X_{t_{k}}-X_{t_{k-1}}\right), \phi_{k, l} \in C_{b, \text { lip }}\left(R^{d \times k}\right)$. We introduce the norm on this space

$$
\|K\|_{H_{G}^{p}\left([0, T] \times R_{0}^{d}\right)}:=\hat{\mathbb{E}}\left[\int_{0}^{T} \sup _{v \in \mathcal{V}} \int_{R_{0}^{d}}|K(u, z)|^{p} v(d z) d u\right]^{\frac{1}{p}}, \quad p=1,2 .
$$

Definition 2.6 Let $0 \leq s<t \leq T$. The Itô integral of $K \in H_{G}^{S}\left([0, T] \times R_{0}^{d}\right)$ w.r.t. jump measure $L$ is defined as

$$
\int_{s}^{t} \int_{R_{0}^{d}} K(u, z) L(d u, d z):=\sum_{s<u \leq t} K\left(u, \Delta X_{u}\right), \quad \text { q.s. }
$$

Lemma 2.2 For every $K \in H_{G}^{S}\left([0, T] \times R_{0}^{d}\right)$, we have that $\int_{0}^{T} \int_{R_{0}^{d}} K(u, z) L(d u, d z)$ is an element of $L_{G}^{2}\left(\Omega_{T}\right)$.

Let $H_{G}^{p}\left([0, T] \times R_{0}^{d}\right)$ denote the topological completion of $H_{G}^{S}\left([0, T] \times R_{0}^{d}\right)$ under the norm $\|\cdot\|_{H_{G}^{p}\left([0, T] \times R_{0}^{d}\right)}, p=1,2$. Then Itô integral can be continuously extended to the whole space $H_{G}^{p}\left([0, T] \times R_{0}^{d}\right), p=1,2$. Moreover, by Lemma 2.2 we know that the extended operator takes value in $L_{G}^{2}\left(\Omega_{T}\right), p=1,2$.

Lemma 2.3 Let $K^{1}(t, z), K^{2}(t, z) \in H_{G}^{2}\left([0, T] \times R_{0}^{d}\right)$. If $K^{1}(t, z) \leq K^{2}(t, z)$ for $t \in[0, T]$, then

$$
\int_{0}^{T} \int_{R_{0}^{d}} K^{1}(t, z) L(d t, d z) \leq \int_{0}^{T} \int_{R_{0}^{d}} K^{2}(t, z) L(d t, d z)
$$

Proof Let $K^{1}(t, z), K^{2}(t, z) \in H_{G}^{S}\left([0, T] \times R_{0}^{d}\right)$, by Definition 2.6, the following holds:

$$
\begin{aligned}
\int_{0}^{T} \int_{R_{0}^{d}} K^{1}(t, z) L(d t, d z) & =\sum_{0<u \leq T} K^{1}\left(u, \Delta X_{u}\right) \\
& \leq \sum_{0<u \leq T} K^{2}\left(u, \Delta X_{u}\right)=\int_{0}^{T} \int_{R_{0}^{d}} K^{2}(t, z) L(d t, d z) .
\end{aligned}
$$

For $K(t, z) \in H_{G}^{p}\left([0, T] \times R_{0}^{d}\right)$, the inequality still holds under a regular argument. 
To consider the solution of G-SDEs, let us introduce the new norm on the integrands: for a process $\eta$, define

$$
\|\eta\|_{\hat{M}_{G}^{p}(0, T)}^{p}:=\int_{0}^{T} \hat{\mathbb{E}}\left[\left|\eta_{t}\right|^{p}\right] d t, \quad p \geq 1
$$

The completion of the space under this norm will be denoted as $\hat{M}_{G}^{p}(0, T)$. Note that

$$
\hat{\mathbb{E}}\left[\int_{0}^{T}\left|\eta_{t}\right|^{p} d t\right] \leq \int_{0}^{T} \hat{\mathbb{E}}\left[\left|\eta_{t}\right|^{p}\right] d t
$$

thus appropriate integrals will be always well defined.

Similarly, we need to adjust the space of integrands for the jump measure. Let $\hat{H}_{G}^{2}([0$, $\left.T] \times R_{0}^{d}\right)$ denote the completion of all $H_{G}^{S}\left([0, T] \times R_{0}^{d}\right)$ under the norm

$$
\|K\|_{\hat{H}_{G}^{2}\left([0, T] \times R_{0}^{d}\right)}^{2}:=\int_{0}^{T} \hat{\mathbb{E}}\left[\sup _{v \in \mathcal{V}} \int_{R_{0}^{d}} K^{2}(u, z) v(d z)\right] d u .
$$

We consider the following G-SDE driven by $d$-dimensional $G$-Brownian motion $B$ and the pure jump G-Lévy process $L$ (in this paper we always use Einstein's convention):

$$
\begin{aligned}
d Y_{t}= & f\left(t, Y_{t}\right) d t+g_{j, k}\left(t, Y_{t}\right) d\left\langle B^{j}, B^{k}\right\rangle_{t}+\sigma_{i}\left(t, Y_{t}\right) d B_{t}^{i} \\
& +\int_{R_{0}^{d}} K\left(t, Y_{t}, z\right) L(d t, d z) .
\end{aligned}
$$

Let $\hat{M}_{G}^{2}\left(0, T ; R^{n}\right)$ denote the space of $R^{n}$-valued process and for each element belong to $\hat{M}_{G}^{2}(0, T)$. We can define the space $\hat{H}_{G}^{2}\left([0, T] \times R_{0}^{d} ; R^{n}\right)$ in a similar way.

Theorem 2.4 ([5]) Suppose that $f(t, x), g_{j, k}(t, x), \sigma_{i}(t, x), K(t, x)$ are Lipschitz continuous w.r.t. $x$ uniformly. For each $x \in R^{n}, f(\cdot, x), g_{j, k}(\cdot, x), \sigma_{i}(\cdot, x) \in \hat{M}_{G}^{2}\left(0, T ; R^{n}\right), K(\cdot, x, \cdot) \in$ $\hat{H}_{G}^{2}\left([0, T] \times R_{0}^{d} ; R^{n}\right)$. Then G-SDE (2.3) with the initial condition $Y_{0} \in R^{n}$ has a unique solution $Y_{t} \in \hat{M}_{G}^{2}\left(0, T ; R^{n}\right)$.

\section{Existence of solution for G-SDEs with discontinuous coefficients}

Definition 3.1 If, for any $0 \leq s \leq t$, the process $U_{t} \in \hat{M}_{G}^{2}\left(0, T ; R^{n}\right)$ satisfies the following inequality:

$$
\begin{aligned}
U_{t} \geq & U_{s}+\int_{s}^{t} f\left(u, U_{u}\right) d u+\int_{s}^{t} g_{j, k}\left(u, U_{u}\right) d\left\langle B^{j}, B^{k}\right\rangle_{u} \\
& +\int_{s}^{t} \sigma_{i}\left(u, U_{u}\right) d B_{u}^{i}+\int_{s}^{t} \int_{R_{0}^{d}} K\left(u, U_{u}, z\right) L(d u, d z),
\end{aligned}
$$

q.s., then it is said to be an upper solution of $G-\operatorname{SDE}(2.3)$ on the interval $[0, T]$. 
Definition 3.2 If, for any $0 \leq s \leq t$, the process $L_{t} \in \hat{M}_{G}^{2}\left(0, T ; R^{n}\right)$ satisfies the following inequality:

$$
\begin{aligned}
L_{t} \leq & L_{s}+\int_{s}^{t} f\left(u, L_{u}\right) d u+\int_{s}^{t} g_{j, k}\left(u, L_{u}\right) d\left\langle B^{j}, B^{k}\right\rangle_{u} \\
& +\int_{s}^{t} \sigma_{i}\left(u, L_{u}\right) d B_{u}^{i}+\int_{s}^{t} \int_{R_{0}^{d}} K\left(u, L_{u}, z\right) L(d u, d z),
\end{aligned}
$$

q.s., then it is said to be a lower solution of $G-\operatorname{SDE}(2.3)$ on the interval $[0, T]$.

Suppose that $U_{t}$ and $L_{t}$ are the respective upper and lower solutions of the G-SDE

$$
\begin{aligned}
d Y_{t}= & f(t, w) d t+g_{j, k}(t, w) d\left\langle B^{j}, B^{k}\right\rangle_{t}+\sigma_{i}\left(t, Y_{t}\right) d B_{t}^{i} \\
& +\int_{R_{0}^{d}} K(t, w, z) L(d t, d z), \quad t \in[0, T], w \in \Omega,
\end{aligned}
$$

where $f(\cdot, w), g_{j, k}(\cdot, w) \in \hat{M}_{G}^{2}\left(0, T ; R^{n}\right), K(\cdot, w, \cdot) \in \hat{H}_{G}^{2}\left([0, T] \times R_{0}^{d} ; R^{n}\right)$ for $w \in \Omega, \sigma_{i}(\cdot, x) \in$ $\hat{M}_{G}^{2}\left(0, T ; R^{n}\right)$ for each $x \in R^{n}$ and $\sigma_{i}(t, x)$ is Lipschitz continuous in $x$. Define two functions $p, q:[0, T] \times R^{n} \times \Omega \rightarrow R^{n}$ by

$$
\begin{aligned}
& p(t, x, w)=\max \left\{L_{t}(w), \min \left\{U_{t}(w), x\right\}\right\}, \\
& q(t, x, w)=p(t, x, w)-x,
\end{aligned}
$$

and consider the following G-SDE:

$$
\begin{aligned}
d Y_{t}=\tilde{f}\left(t, Y_{t}\right) d t+\tilde{g}_{j, k}\left(t, Y_{t}\right) d\left\langle B^{j}, B^{k}\right\rangle_{t}+\tilde{\sigma}_{i}\left(t, Y_{t}\right) d B_{t}^{i} \\
+\int_{R_{0}^{d}} \tilde{K}\left(t, Y_{t}, z\right) L(d t, d z), \quad t \in[0, T]
\end{aligned}
$$

with a given constant initial condition $Y_{0} \in R^{n}$, where

$$
\begin{aligned}
& \tilde{f}(t, x, w)=f(t, w)+q(t, x, w), \\
& \tilde{g}_{j, k}(t, x, w)=g_{j, k}(t, w)+q(t, x, w), \\
& \tilde{\sigma}_{i}(t, x, w)=\sigma_{i}(t, p(t, x, w)), \\
& \tilde{K}(t, x, z)=K(t, w, z)+q(t, x, w) .
\end{aligned}
$$

It is clear that $\tilde{f}, \tilde{g}_{j, k}, \tilde{\sigma}_{i}, \tilde{K}$ are Lipschitz continuous in $x$, thus the conditions of Theorem 2.4 are satisfied. Then G-SDE (3.5) has a unique solution $Y_{t} \in \hat{M}_{G}^{2}\left(0, T ; R^{n}\right)$.

Lemma 3.1 Suppose that $U_{t}$ and $L_{t}$ are the respective upper and lower solutions of G-SDE (3.3) satisfying $L_{t} \leq U_{t}$ for $t \in[0, T]$. Then $U_{t}$ and $L_{t}$ are the upper and lower solutions of G-SDE (3.5), respectively. 
Proof For $0 \leq s \leq t$, we have

$$
\begin{aligned}
U_{s}+ & \int_{s}^{t} \tilde{f}\left(u, U_{u}\right) d u+\int_{s}^{t} \tilde{g}_{j, k}\left(u, U_{u}\right) d\left\langle B^{j}, B^{k}\right\rangle_{u} \\
& +\int_{s}^{t} \tilde{\sigma}_{i}\left(u, U_{u}\right) d B_{u}+\int_{s}^{t} \int_{R_{0}^{d}} \tilde{K}\left(u, U_{u}, u\right) L(d u, d z) \\
= & U_{s}+\int_{s}^{t}\left[f(u, w)+q\left(u, U_{u}, w\right)\right] d u+\int_{s}^{t}\left[g_{j, k}(u, w)+q\left(u, U_{u}, w\right)\right] d\left\langle B^{j}, B^{k}\right\rangle_{u} \\
& +\int_{s}^{t} \sigma_{i}\left(u, p\left(u, U_{u}, w\right)\right) d B_{u}^{i}+\int_{s}^{t} \int_{R_{0}^{d}}\left[K(u, w, z)+q\left(u, U_{u}, w\right)\right] L(d u, d z) \\
= & U_{s}+\int_{s}^{t} f(u, w) d u+\int_{s}^{t} g_{j, k}(u, w) d\left\langle B^{j}, B^{k}\right\rangle_{u} \\
& +\int_{s}^{t} \sigma_{i}\left(u, U_{u}\right) d B_{u}^{i}+\int_{s}^{t} \int_{R_{0}^{d}} K(u, w, z) L(d u, d z) \\
\leq & U_{t},
\end{aligned}
$$

where we have used $p\left(t, U_{t}, w\right)=U_{t}$ and $q\left(t, U_{t}, w\right)=0$. Therefore $U_{t}$ is an upper solution of G-SDE (3.5). One can show that $L_{t}$ is a lower solution of G-SDE (3.5) in a similar way as above.

By Lemma 3.1 we know that if $U_{t}$ and $L_{t}$ are upper and lower solutions of G-SDE (3.3), then they are the respective upper and lower solutions for G-SDE (3.5). Suppose that $Y_{t}$ is the solution of G-SDE (3.5) such that

$$
L_{t}<Y_{t}<U_{t}, \quad t \in[0, T] \text {, q.s. }
$$

Since $p\left(t, Y_{t}, w\right)=Y_{t}, q\left(t, Y_{t}, w\right)=0$, then

$$
\begin{aligned}
d Y_{t}= & \tilde{f}\left(t, Y_{t}\right) d t+\tilde{g}_{j, k}\left(t, Y_{t}\right) d\left\langle B^{j}, B^{k}\right\rangle_{t}+\tilde{\sigma}_{i}\left(t, Y_{t}\right) d B_{t}^{i}+\int_{R_{0}^{d}} \tilde{K}\left(t, Y_{t}, z\right) L(d t, d z) \\
= & {\left[f(t, w)+q\left(t, Y_{t}, w\right)\right] d t+\left[g_{j, k}(t, w)+q\left(u, Y_{t}, w\right)\right] d\left\langle B^{j}, B^{k}\right\rangle_{t} } \\
& +\sigma_{i}\left(t, p\left(t, Y_{t}, w\right)\right) d B_{t}^{i}+\int_{R_{0}^{d}}\left[K(t, w, z)+q\left(t, Y_{t}, w\right)\right] L(d t, d z) \\
= & f(t, w) d t+g_{j, k}(t, w) d\left\langle B^{j}, B^{k}\right\rangle_{t}+\sigma_{i}\left(t, Y_{t}\right) d B_{t}^{i}+\int_{R_{0}^{d}} K(t, w, z) L(d t, d z),
\end{aligned}
$$

which implies that $Y_{t}$ is a solution of G-SDE (3.3). Thus, if we can show that any solution $Y_{t}$ of problem (3.5) does satisfy inequality (3.8), then $Y_{t}$ is also the solution of G-SDE (3.3).

\section{Theorem 3.1 Suppose that}

(i) $f(\cdot, w), g_{j, k}(\cdot, w) \in \hat{M}_{G}^{2}\left(0, T ; R^{n}\right), K(\cdot, w, \cdot) \in \hat{H}_{G}^{2}\left([0, T] \times R_{0}^{d} ; R^{n}\right)$ for $w \in \Omega$, $\sigma_{i}(\cdot, x) \in \hat{M}_{G}^{2}\left(0, T ; R^{n}\right)$ for each $x \in R^{n}$ and $\sigma(t, x)$ is Lipschitz continuous in $x$;

(ii) the respective upper and lower solutions $U_{t}$ and $L_{t}$ of G-SDE (3.3) satisfy $L_{t}<U_{t}$ for $t \in[0, T]$ 
(iii) $Y_{0} \in R^{n}$ is a given initial value with $\hat{\mathbb{E}}\left[\left|Y_{0}\right|^{2}\right]<\infty$ and $L_{0}<Y_{0}<U_{0}$.

Then there exists a unique solution $Y_{t} \in \hat{M}_{G}^{2}\left(0, T ; R^{n}\right)$ of G-SDE (3.3) such that $L_{t}<Y_{t}<U_{t}$ for $t \in[0, T]$, q.s.

Proof We only need to prove that the solution $Y_{t}$ of G-SDE (3.5) does satisfy inequality (3.8). Assume that there exists an arbitrary interval $\left(t_{1}, t_{2}\right) \subset[0, T]$ such that $Y_{t_{1}}=L_{t_{1}}$ and $Y_{t}<L_{t}$ for $t \in\left(t_{1}, t_{2}\right)$, then we have

$$
\begin{aligned}
Y_{t}-L_{t} \geq & \int_{t_{1}}^{t} \tilde{f}\left(u, Y_{u}\right) d u+\int_{t_{1}}^{t} \tilde{g}_{j, k}\left(u, Y_{u}\right) d\left\langle B^{j}, B^{k}\right\rangle_{u} \\
& +\int_{t_{1}}^{t} \tilde{\sigma}_{i}\left(u, Y_{u}\right) d B_{u}^{i}+\int_{t_{1}}^{t} \int_{R_{0}^{d}} \tilde{K}\left(u, Y_{u}, z\right) L(d u, d z) \\
& -\int_{t_{1}}^{t} \tilde{f}\left(u, L_{u}\right) d u-\int_{t_{1}}^{t} \tilde{g}_{j, k}\left(u, L_{u}\right) d\left\langle B^{j}, B^{k}\right\rangle_{u} \\
& -\int_{t_{1}}^{t} \tilde{\sigma}_{i}\left(u, L_{u}\right) d B_{u}^{i}-\int_{t_{1}}^{t} \int_{R_{0}^{d}} \tilde{K}\left(u, L_{u}, z\right) L(d u, d z) \\
= & \int_{t_{1}}^{t}\left[f(u, w)+q\left(u, Y_{u}, w\right)\right] d u+\int_{t_{1}}^{t}\left[g_{j, k}(u, w)+q\left(u, Y_{u}, w\right)\right] d\left\langle B^{j}, B^{k}\right\rangle_{u} \\
& +\int_{t_{1}}^{t} \sigma_{i}\left(u, p\left(u, Y_{u}, w\right)\right) d B_{u}^{i} \\
& +\int_{t_{1}}^{t} \int_{R_{0}^{d}}\left[K(u, z)+q\left(u, Y_{u}, w\right)\right] L(d u, d z)-\int_{t_{1}}^{t}\left[f(u, w)+q\left(u, L_{u}, w\right)\right] d u \\
& -\int_{t_{1}}^{t}\left[g_{j, k}(u, w)+q\left(u, L_{u}, w\right)\right] d\left\langle B^{j}, B^{k}\right\rangle_{u}-\int_{t_{1}}^{t} \sigma_{i}\left(u, p\left(u, L_{u}, w\right)\right) d B_{u}^{i} \\
& -\int_{t_{1}}^{t} \int_{R_{0}^{d}}\left[K(u, z)+q\left(u, L_{u}, w\right)\right] L(d u, d z) .
\end{aligned}
$$

Since $Y_{t} \leq L_{t} \leq U_{t}$ for $t \in\left(t_{1}, t_{2}\right)$, then $p\left(t, L_{t}, w\right)=L_{t}$ and $p\left(t, Y_{t}, w\right)=L_{t}$. Also $q\left(t, L_{t}, w\right)=0$ and $q\left(t, Y_{t}, w\right)=L_{t}-Y_{t}$. Thus

$$
\begin{aligned}
Y_{t}-L_{t} \geq & \int_{t_{1}}^{t}\left(L_{u}-Y_{u}\right) d u+\int_{t_{1}}^{t}\left(L_{u}-Y_{u}\right) d\left\langle B^{j}, B^{k}\right\rangle_{u} \\
& +\int_{t_{1}}^{t} \int_{R_{0}^{d}}\left(L_{u}-Y_{u}\right) L(d u, d z)>0,
\end{aligned}
$$

which yields a contradiction. Thus $Y_{t} \geq L_{t}$ for $t \in[0, T]$. By using similar arguments as above, one can show that $Y_{t} \leq U_{t}$ for $t \in[0, T]$. Thus the proof is finished.

Now we consider the following G-SDE:

$$
\begin{aligned}
d Y_{t}= & f\left(t, Y_{t}\right) d t+g_{j, k}\left(t, Y_{t}\right) d\left\langle B^{j}, B^{k}\right\rangle_{t} \\
& +\sigma_{i}\left(t, Y_{t}\right) d B_{t}^{i}+\int_{R_{0}^{d}} K\left(t, Y_{t}, z\right) L(d t, d z),
\end{aligned}
$$


where $f(t, x), g_{j, k}(t, x)$ and $K(t, x, z)$ do not need to be Lipschitz continuous with respect to $x$, only $\sigma_{i}(t, x)$ is Lipschitz continuous in $x$.

\section{Theorem 3.2 Suppose that}

(i) for each $x \in R^{n}, f(\cdot, x), g_{j, k}(\cdot, x), \sigma_{i}(\cdot, x) \in \hat{M}_{G}^{2}\left(0, T ; R^{n}\right), K(\cdot, x, \cdot) \in \hat{H}_{G}^{2}\left([0, T] \times R_{0}^{d} ; R^{n}\right)$;

(ii) $\sigma_{i}(t, x)$ is Lipschitz continuous in $x, f(t, x), g_{j, k}(t, x)$ and $K(t, x, z)$ are increasing in $x$;

(iii) $U_{t}$ and $L_{t}$ are the respective upper and lower solutions of G-SDE (3.12). Moreover, $K\left(\cdot, U_{t}, \cdot\right), K\left(\cdot, L_{t}, \cdot\right) \in \hat{H}_{G}^{2}\left([0, T] \times R_{0}^{d} ; R^{n}\right)$ and $L_{t} \leq U_{t}$ for $t \in[0, T]$.

Then there exists at least one solution $Y_{t} \in \hat{M}_{G}^{2}\left(0, T ; R^{n}\right)$ of $G-S D E(3.12)$ such that $L_{t} \leq$ $Y_{t} \leq U_{t}$ for $t \in[0, T]$, q.s.

Proof Denote the order interval $[L, U]$ in $\hat{M}_{G}^{2}\left(0, T ; R^{n}\right)$ by $\mathcal{H}$, that is, $\mathcal{H}=\{Y: Y \in$ $\hat{M}_{G}^{2}\left(0, T ; R^{n}\right)$ and $\left.L_{t} \leq Y_{t} \leq U_{t}\right\}$ for $t \in[0, T]$, which is closed and bounded. By using the monotone convergence theorem in [5], one can prove the convergence of a monotone sequence that belongs to $\mathcal{H}$ in $\hat{M}_{G}^{2}\left(0, T ; R^{n}\right)$. Thus $\mathcal{H}$ is a regularly ordered metric space with the norm of $\hat{M}_{G}^{2}\left(0, T ; R^{n}\right)$.

Since $f(t, x), g_{j, k}(t, x)$ and $K(t, x, z)$ are increasing in $x$, it is easy to see that for any process $V \in \mathcal{H}, U_{t}$ and $L_{t}$ are the respective upper and lower solutions for the G-SDE

$$
d Y_{t}=f\left(t, V_{t}\right) d t+g_{j, k}\left(t, V_{t}\right) d\left\langle B^{j}, B^{k}\right\rangle_{t}+\sigma_{i}\left(t, Y_{t}\right) d B_{t}^{i}+\int_{R_{0}^{d}} K\left(t, V_{t}, z\right) L(d t, d z) .
$$

Hence, by Theorem 3.1, for any $Y_{0} \in R^{n}$ with $\hat{\mathbb{E}}\left[\left|Y_{0}\right|^{2}\right]<\infty$, and $L_{0} \leq Y_{0} \leq U_{0}$, G-SDE (3.13) has a unique solution $Y_{t} \in \hat{M}_{G}^{2}\left(0, T ; R^{n}\right)$ such that $L_{t} \leq Y_{t} \leq U_{t}$ for $t \in[0, T]$, q.s.

Define an operator $F: \mathcal{H} \rightarrow \mathcal{H}$ by $F(V)=Y$, where $Y$ is the unique solution of $G$-SDE (3.13). For all $t \in[0, T]$, let $V_{t}^{1}, V_{t}^{2} \in \mathcal{H}$ and $V_{t}^{1} \leq V_{t}^{2}$ and define $Y_{t}^{1}=F\left(V_{t}^{1}\right), Y_{t}^{2}=F\left(V_{t}^{2}\right)$. Since $f, g, K$ are increasing functions, then

$$
\begin{aligned}
Y_{t}^{1}= & F\left(V_{t}^{1}\right) \\
= & Y_{0}+\int_{0}^{t} f\left(u, V_{u}^{1}\right) d u+\int_{0}^{t} g_{j, k}\left(t, V_{u}^{1}\right) d\left\langle B^{j}, B^{k}\right\rangle_{u}+\int_{0}^{t} \sigma_{i}\left(t, Y_{t}^{1}\right) d B_{t}^{i} \\
& +\int_{0}^{t} \int_{R_{0}^{d}} K\left(t, V_{t}^{1}, z\right) L(d t, d z) \\
\leq & Y_{0}+\int_{0}^{t} f\left(u, V_{u}^{2}\right) d u+\int_{0}^{t} g_{j, k}\left(t, V_{u}^{2}\right) d\left\langle B^{j}, B^{k}\right\rangle_{u}+\int_{0}^{t} \sigma_{i}\left(t, Y_{t}^{1}\right) d B_{t}^{i} \\
& +\int_{0}^{t} \int_{R_{0}^{d}} K\left(t, V_{t}^{2}, z\right) L(d t, d z),
\end{aligned}
$$

which implies that $Y_{t}^{1}$ is a lower solution of the G-SDE

$$
\begin{aligned}
Y_{t}= & Y_{0}+\int_{0}^{t} f\left(u, V_{u}^{2}\right) d u+\int_{0}^{t} g_{j, k}\left(t, V_{u}^{2}\right) d\left\langle B^{j}, B^{k}\right\rangle_{u}+\int_{0}^{t} \sigma_{i}\left(t, Y_{t}\right) d B_{t}^{i} \\
& +\int_{0}^{t} \int_{R_{0}^{d}} K\left(t, V_{t}^{2}, z\right) L(d t, d z) .
\end{aligned}
$$

However, this problem has an upper solution $U_{t}$. Then the solution of G-SDE (3.15) $Y_{t}^{2}$ satisfies $Y_{t}^{1} \leq Y_{t}^{2} \leq U_{t}$. Hence $F$ is an increasing mapping and, by Theorem 3.3, it has a 
fixed point $Y^{*}=F\left(Y^{*}\right) \in \mathcal{H}$ such that $L_{t} \leq Y_{t}^{*} \leq U_{t}$, q.s. and

$$
\begin{aligned}
Y_{t}^{*}= & Y_{0}+\int_{0}^{t} f\left(u, Y_{t}^{*}\right) d u+\int_{0}^{t} g_{j, k}\left(t, Y_{t}^{*}\right) d\left\langle B^{j}, B^{k}\right\rangle_{u}+\int_{0}^{t} \sigma_{i}\left(t, Y_{t}^{*}\right) d B_{t}^{i} \\
& +\int_{0}^{t} \int_{R_{0}^{d}} K\left(t, Y_{t}^{*}, z\right) L(d t, d z) .
\end{aligned}
$$

Thus the proof is finished.

Example 3.1 Consider the following scalar stochastic differential equation:

$$
d Y_{t}=H\left(Y_{t}\right) d t+\left\{Y_{t}\right\} d\langle B\rangle_{t}+d B_{t}+\int_{R_{0}^{d}}\left\{Y_{t}\right\} L(d t, d z)
$$

where the Heaviside function $H: R \rightarrow R$ is defined by

$$
H(x)= \begin{cases}0, & \text { if } x<0 \\ 1, & \text { if } x \geq 0\end{cases}
$$

This is an important function in science, and it is considered to be a fundamental function in engineering. The fractional part function $\{x\}: R \rightarrow[0,1)$ has discontinuities at the integers and is defined by

$$
\{x\}=x-[x], \quad x \in R,
$$

where $[x]$ is the floor function. The importance of this function is clear from the sawtooth waves which are used in music and computer graphics.

Let $U_{t}=U_{0}+\int_{0}^{t} d u+\int_{0}^{t} d\langle B\rangle_{u}+\int_{0}^{t} d B_{u}+\int_{0}^{t} \int_{R_{0}} L(d u, d z)$ for $t \in[0, T]$. Then we have

$$
\begin{aligned}
U_{t} & =U_{0}+\int_{0}^{t} d u+\int_{0}^{t} d\langle B\rangle_{u}+\int_{0}^{t} d B_{u}+\int_{0}^{t} \int_{R_{0}} L(d u, d z) \\
& =U_{s}+\int_{s}^{t} d u+\int_{s}^{t} d\langle B\rangle_{u}+\int_{s}^{t} d B_{u}+\int_{s}^{t} \int_{R_{0}} L(d u, d z) \\
& \geq U_{s}+\int_{s}^{t} H\left(U_{u}\right) d u+\int_{s}^{t}\left\{U_{u}\right\} d\langle B\rangle_{u}+\int_{s}^{t} d B_{u}+\int_{s}^{t} \int_{R_{0}}\left\{U_{u}\right\} L(d u, d z),
\end{aligned}
$$

where $U_{s}=U_{0}+\int_{0}^{s} d u+\int_{0}^{s} d\langle B\rangle_{u}+\int_{0}^{s} d B_{u}+\int_{0}^{s} \int_{R_{0}} L(d u, d z)$ for $0 \leq s \leq t \leq T$. This implies that $U_{t}$ is the upper solution of equation (3.17). In a similar way, one can show that $L_{t}=$ $L_{0}+\int_{0}^{t} d B_{u}$ is a lower solution of equation (3.17). Then, by Theorem 3.2, there exists at least one solution for equation (3.17).

For the following definition and theorem, see [14].

Definition 3.3 An ordered metric space $M$ is called regularly (resp. fully regularly) ordered if each monotone and order (resp. metrically ) bounded ordinary sequence of $M$ converges. 
Theorem 3.3 If $[a, b]$ is a non-empty order interval in a regularly ordered metric space, then each increasing mapping $F:[a, b] \rightarrow[a, b]$ has the least and the greatest fixed points.

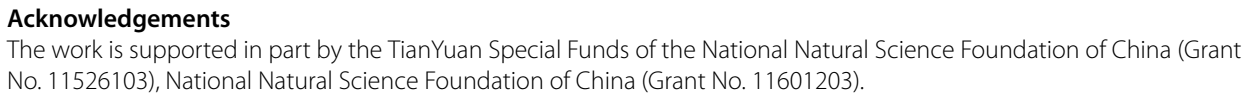

\section{Competing interests}

The authors declare that they have no competing interests.

Authors' contributions

Both of the authors jointly worked on deriving the results and approved the final manuscript.

\section{Author details}

${ }^{1}$ School of Mathematical Science, Nanjing Normal University, Nanjing, 210023, P.R. China. ${ }^{2}$ Jinling Institute of Technology, Nanjing, 211169, P.R. China. ${ }^{3}$ Jinling College, Nanjing University, Nanjing, 210089, P.R. China.

\section{Publisher's Note}

Springer Nature remains neutral with regard to jurisdictional claims in published maps and institutional affiliations.

Received: 6 March 2017 Accepted: 13 June 2017 Published online: 30 June 2017

\section{References}

1. Peng, S: Filtration consistent nonlinear expectations and evaluations of contingent claims. Acta Math. Appl. Sinica (Engl. Ser.) 20, 1-24 (2004)

2. Peng, S: G-Expectations, G-Brownian motion and related stochastic calculus of Itô's type. In: Benth, FE, Di Nunno, G, Lindstrom, T, Øksendal, B, Zhang, T (eds.) Proceedings of the 2005 Abel Symposium, pp. 541-567. Springer, Berlin (2006). Preprint version in: arXiv:math/0601035v2

3. Hu, M, Peng, S: G-Lévy processes under sublinear expectations. arXiv:0911.3533v1

4. Ren, L: On representation theorem of sublinear expectation related to G-Lévy processes and paths of G-Lévy processes. Stat. Probab. Lett. 83, 1301-1310 (2013)

5. Paczka, K: Itô calculus and jump diffusions for G-Lévy processes. arXiv:1211.2973v3

6. Faizullah, F, Piao, D: Existence of solutions for G-SDEs with upper and lower solutions in the reverse order. Int. J. Phys. Sci. 7, 432-439 (2012)

7. Faizullah, F: Existence of solutions for stochastic differential equations under G-Brownian motion with discontinuous coefficients. Z. Naturforsch. A 67, 692-698 (2012)

8. Paczka, K: G-Martingale representation in the G-Lévy setting. arXiv:1404.2121v1

9. Denis, L, Hu, M, Peng, S: Function spaces and capacity related to a sublinear expectation: application to G-Brownian motion paths. Potential Anal. 34, 139-161 (2011)

10. Soner, M, Touzi, N, Zhang, J: Martingale representation theorem for the G-expectation. Stoch. Process. Appl. 121, 265-287 (2011)

11. Peng, S: Multi-dimensional G-Brownian motion and related stochastic calculus under G-expectation. Stoch. Process. Appl. 118, 2223-2253 (2008)

12. Peng, S: Nonlinear expectations and stochastic calculus under uncertainly. arXiv:1002.4546v1 (2010)

13. Li, X, Peng, S: Stopping times and related Itô's calculus with G-Brownian motion. Stoch. Process. Appl. 121, 1492-1508 (2011)

14. Heikkila, S, Hu, S: On fixed points of multifunctions in ordered spaces. Appl. Anal. 51, 115-127 (1993)

\section{Submit your manuscript to a SpringerOpen ${ }^{\circ}$ journal and benefit from:}

- Convenient online submission

- Rigorous peer review

- Open access: articles freely available online

- High visibility within the field

- Retaining the copyright to your article

Submit your next manuscript at $\boldsymbol{\text { springeropen.com }}$ 